\title{
Visualização de informações para acompanhamento de alunos em ambientes de aprendizagem eletrônica
}

\author{
Maylon Pires Macedo ${ }^{1}$, Luciana A. M. Zaina ${ }^{1}$ \\ ${ }^{1}$ Universidade Federal de São Carlos - Campus Sorocaba \\ Pesquisa em andamento com início em Setembro de 2018
}

\begin{abstract}
Resumo. A área de Visualização da Informação estuda formas de apresentar dados graficamente para auxiliar a tomada de decisão. InfoVis é um framework da área de Visualização da Informação para elaborar visualizações direcionadas por dados e tarefas executadas pelo usuário. O objetivo deste projeto é propor recomendações, baseadas no InfoVis, para auxiliar o desenvolvimento de visualizações voltadas a tomadores de decisão em aplicações e-learning. Com as recomendações propostas espera-se contribuir no processo de elaboração de visualizações, tornando melhor a compreensão sobre os dados e resultando em visualizações mais aderentes às necessidades do público-alvo dentro do contexto educacional.
\end{abstract}

\section{Introdução}

Nos últimos anos os profissionais da educação tem sido favoráveis a inclusão de visualização da informação para melhorar as análises do trabalho desenvolvido [Carvalho et al. 2016, Rigo et al. 2014, Reyes 2015]. Ferramentas que não utilizam metodologias para desenvolver visualizações podem agregar uma má experiência ao usuário em sua utilização [Reyes 2015, Angeli et al. 2017, Padilha e Souza 2017]. A [ISO/IEC 2001] define experiência do usuário ( $\mathrm{UX}^{1}$ ) como sendo a percepção sobre o resultado do uso de um produto, sistema ou serviço.

Os dados gerados pelas interações dos alunos com ambientes de ensino são categorizados pela literatura como "dados educacionais" [Jordão et al. 2014, Vieira et al. 2018, Moissa et al. 2015], e podem compreender informações como: notas, dados demográficos, atividades realizadas, entre outras [Tervakari et al. 2014, Barros et al. 2017]. Por mais que estes dados possam ser analisados individualmente em tabelas planas, este tipo de visualização não permite inferir padrões, encontrar tendências ou valores discrepantes. Dificultando a interpretação dos dados e, por consequência, a tomada de decisão [Guimar 2014].

[Padilha e Souza 2017] relatam a experiência de professores aplicando ferramentas da área de negócios para analisar dados educacionais. Os autores conseguiram realizar a análise desejada, porém foi necessário um esforço alto para operar as ferramentas, pois dados educacionais são volumosos e não trazem informações relevantes em sua forma pura, sendo necessário ainda algum tratamento visual [Majumdar e Iyer 2016].

Para guiar o desenvolvimento de visualizações que considerem às tarefas, dados e domínio do usuário surgiu o framework proposto por [Munzner 2014], denominado

\footnotetext{
${ }^{1}$ do inglês, User eXperience.
} 
VIII Congresso Brasileiro de Informática na Educação (CBIE 2019)

Anais dos Workshops do VIII Congresso Brasileiro de Informática na Educação (WCBIE 2019)

InfoVis. O objetivo é conduzir o designer a fazer escolhas que sejam adequadas ao contexto do usuário para elaborar o idioma da visualização ${ }^{2}$. Estudos fornecem relatos demonstrando resultados positivos da aplicação de conceitos semelhantes aos do InfoVis (proposto por [Munzner 2014]) no desenvolvimento de visualizações sobre dados educacionais [Majumdar e Iyer 2016, Jordão et al. 2014].

O problema de pesquisa abordado por este trabalho é a falta de aplicação de métodos de Interação Humano Computador (IHC) no desenvolvimento de visualizações de dados educacionais. Os resultados deste serão recomendações focadas em usabilidade para a construção de visualizações adequadas à auxiliar profissionais da educação na tarefa de análise dos dados.

\section{Objetivos}

A questão de pesquisa norteadora deste projeto é: Como os métodos de IHC tem sido trabalhados pelos pesquisadores durante o desenvolvimento de visualizações de dados no contexto educacional?. O objetivo é gerar e reunir evidências que permitam propor recomendações que possam dar suporte ao desenvolvimento de visualizações levando em conta a usabilidade neste contexto.

\section{Metodologia}

A metodologia de [Rusu et al. 2011] será aplicada para nortear a elaboração das recomendações que serão propostas por esta pesquisa. [Rusu et al. 2011] define um framework contendo seis etapas para guiar o desenvolvimento de recomendações baseadas em usabilidade. A metodologia é voltada para contextos específicos onde as heurísticas tradicionais podem não ser suficientes.

As seis etapas definidas por [Rusu et al. 2011] são: Estágio Exploratório: estudo da bibliografia sobre o tema de pesquisa especifico para coletar informações sobre características relacionadas a usabilidade; Estágio Descritivo: formalizar conceitos principais destacando as características mais importantes sobre o contexto; Estágio Correlacional: identificar as características de usabilidade que deveriam ser necessárias baseado em heurísticas tradicionais e análises levando em conta o contexto da aplicação; Estágio Explicativo: definir formalmente o conjunto de propostas; Estágio de Validação: validar a proposta por meio de avaliações com especialistas ou testes com usuário; Estágio de Refinamento: refinar a proposta com base nas informações coletadas na etapa de validação.

A metodologia definida para guiar o desenvolvimento do projeto de mestrado foi dividida em etapas. A Figura 1 representa a relação entre as etapas propostas por [Rusu et al. 2011] e as etapas definidas para guiar o desenvolvimento da pesquisa, que foram definidas da seguinte forma:

Etapa A - Estudo dos Fundamentos: Realização de um estudo bibliográfico sobre os principais conceitos tangentes ao projeto com a intenção de agregar uma visão geral sobre: visualização de dados educacionais; visualização da informação; InfoVis (por [Munzner 2014]); usabilidade; e UX. O resultado desta etapa será uma revisão sobre o estado da arte, esta atividade será contínua durante todo o projeto já que novas e importantes referências podem surgir.

\footnotetext{
${ }^{2}$ Idioma da visualização pode ser entendido como a maneira que a visualização comunica o usuário sobre os dados através de formatos, cores ou interações.
} 
VIII Congresso Brasileiro de Informática na Educação (CBIE 2019)

Anais dos Workshops do VIII Congresso Brasileiro de Informática na Educação (WCBIE 2019)

Etapa B - Mapeamento Sistemático da Literatura (MSL): Um MSL foi conduzido para investigar trabalhos relacionados ao desenvolvimento de visualizações de dados educacionais. O intuito foi mapear quais metodologias têm sido utilizadas neste contexto e quais são as etapas realizadas durante o desenvolvimento. O MSL seguiu os fundamentos e premissas de [Petersen et al. 2008]. Além de cumprir o intuito principal, o MSL auxiliou na tarefa de encontrar recomendações de desenvolvimento, problemas conhecidos e casos de sucesso, gerando evidências para estabelecer uma relação entre as características apontadas pela literatura e as ações realizadas nos trabalhos.

Etapa C - Extração e consolidação das descobertas: Com base nos artigos encontrados durante o MSL, foram extraídas recomendações para construção de visualizações. A extração foi realizada de maneira categorizada, sendo organizada por: nome do artigo, recorte do texto, qual é o tipo da recomendação (específica ou geral), qual a base da recomendação (percepção humana ou semântica dos dados), informações sobre o stakeholder e uma reflexão. A reflexão serve para situar as escolhas no cenário de uso, elucidando quais eram as condições, intenções e resultados no momento da descoberta. Após a extração realizada pelos autores, as recomendações foram validadas por um trio de especialistas com experiência em IHC, visualização da informação e mineração de dados educacionais.

Etapa D - Elaborar as recomendações: Comparar as recomendações extraídas da literatura com o framework de [Munzner 2014], para delinear a proposta das recomendações que possam apoiar o desenvolvimento de visualizações sobre dados educacionais. As recomendações atuarão de maneira a aliar os aspectos do domínio do usuário sobre os dados e tarefas aos aspectos funcionais da visualização com o intuito de melhorar a usabilidade proporcionada pela ferramenta.

Etapa E - Avaliação da proposta: Se dará por meio de questionário próprio, onde usuários (professores, coordenadores, etc) irão opinar sobre as recomendações e seus resultados. Estará disponível no questionário: o texto da recomendação, seu contexto e um protótipo exemplificando a aplicação da recomendação.

Etapa F - Divulgação dos resultados: Os resultados serão divulgados por meio de artigos científicos e dissertação. Alguns potenciais veículos para publicação dos resultados são: Brazilian Journal of Computers in Education (RBIE); Simpósio Brasileiro Sobre Fatores Humanos em Sistemas Computacionais, entre outros.

$\mathrm{Na}$ ocasião deste artigo a pesquisa situa-se na Etapa $\mathbf{C}$ com a realização da validação com especialistas. O MSL (Etapa B) contou com 1207 artigos provenientes de 5 repositórios de pesquisa e está sendo preparado para ser submetido a um veículo internacional. Os próximos passos são a comparação das extrações com o framework de [Munzner 2014] para gerar as recomendações e executar a avaliação com os usuários.

\section{Resultados esperados}

A execução do MSL não retornou trabalhos com a utilização de metodologias para o desenvolvimento de visualizações que levem em conta métodos de IHC. Os resultados apresentaram uma tendência em manter o foco nas características técnicas de programação e banco de dados, indicando uma lacuna de pesquisa em relação a interação do usuário na visualização de dados educacionais. Como uma das contribuições deste projeto destaca- 
VIII Congresso Brasileiro de Informática na Educação (CBIE 2019)

Anais dos Workshops do VIII Congresso Brasileiro de Informática na Educação (WCBIE 2019)

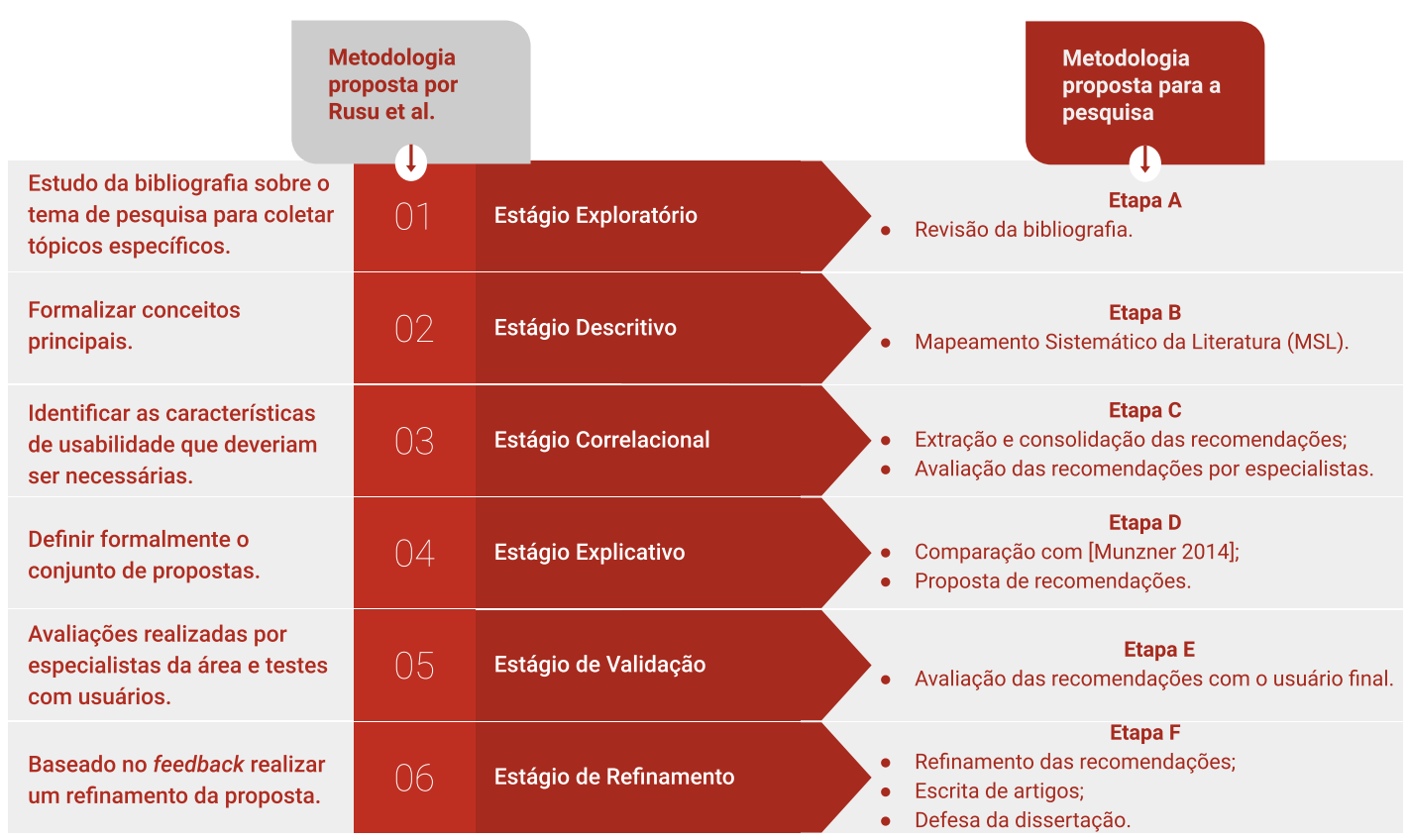

Figura 1. Metodologia utilizada na elaboração das recomendações para desenvolvimento de visualizações sobre dados educacionais.

se a investigação do estado da arte, por meio de estudos sistemáticos, sobre a utilização de técnicas e métodos de IHC no contexto abordado.

Ao fim deste projeto de mestrado é esperado a criação de um catálogo de recomendações para o desenvolvimento de visualizações, dentro do contexto educacional, levando em conta os aspectos de usabilidade. As recomendações serão extraídas de trabalhos onde houveram validações com o usuário, além das recomendações será extraído também uma descrição do contexto de uso (Ex. público-alvo, cenário, problemas relacionados). Após a consolidação, as recomendações serão validadas por especialistas e comparadas com oframework de [Munzner 2014], para dar origem ao conjunto final de recomendações.

\section{Agradecimentos}

O presente trabalho foi realizado com apoio da Coordenação de Aperfeiçoamento de Pessoal de Nível Superior - Brasil (CAPES) - Código de Financiamento 001. Contou com a participação da Prof ${ }^{a}$. Dr ${ }^{a}$. Isabela Gasparini, da Universidade do Estado de Santa Catarina, que agregou com sua experiência sobre estudos sistemáticos e Visualização da Informação em ambientes e-learning. Houve também participação do Prof. Dr. Ranilson Oscar Araújo Paiva, da Universidade Federal de Alagoas, que agregou com sua experiência sobre mineração e visualização de dados educacionais.

\section{Referências}

Angeli, C., Howard, S. K., Ma, J., Yang, J., e Kirschner, P. A. (2017). Data mining in educational technology classroom research: can it make a contribution? Computers and Education, 113:226-242.

Barros, T., Silva, I., e Guedes, L. (2017). Modelagem e visualização científica de dados educacionais: estudo de caso sobre o desempenho em componentes curriculares. In 
VIII Congresso Brasileiro de Informática na Educação (CBIE 2019)

Anais dos Workshops do VIII Congresso Brasileiro de Informática na Educação (WCBIE 2019)

Anais dos Workshops do VI Congresso Brasileiro de Informática na Educação (WCBIE 2017), pages 654-663, Recife, PE. RBIE.

Carvalho, M. J. S., Neves, B., e Melo, R. (2016). Plataforma cultiveduca. In Anais dos Workshops do V Congresso Brasileiro de Informática na Educação (CBIE 2016), pages 134-141, Ubêrlandia, MG. RBIE.

Guimar, C. (2014). Visualização de informação: introdução e influências de IHC. Simpósio Brasileiro sobre Fatores Humanos em Sistemas Computacionais - IHC 2014, pages $79-107$.

ISO/IEC (2001). ISO/IEC 9126. Software engineering - Product quality. ISO/IEC.

Jordão, V., Gonçalves, D., e Gama, S. (2014). Eduvis: Visualizing educational information. In Proceedings of the 8th Nordic Conference on Human-Computer Interaction: Fun, Fast, Foundational, NordiCHI '14, pages 1011-1014, Helsinki, Finland. ACM.

Majumdar, R. e Iyer, S. (2016). iSAT: a visual learning analytics tool for instructors. Research and Practice in Technology Enhanced Learning, 11(16).

Moissa, B., Gasparini, I., e Kemczinski, A. (2015). Educational data mining versus learning analytics: estamos reinventando a roda? um mapeamento sistemático. In Anais do XXVI Simpósio Brasileiro de Informática na Educação (SBIE 2015), pages 11671176, Maceió, Alagoas. RBIE.

Munzner, T. (2014). Visualization analysis and design. A.K. Peters visualization series. A K Peters.

Padilha, T. e Souza, I. (2017). Uma experiência do uso de ferramentas de learning analytics para análise de interações. In Anais dos Workshops do VI Congresso Brasileiro de Informática na Educação (WCBIE 2017), pages 644-653, Recife, PE. RBIE.

Petersen, K., Feldt, R., Mujtaba, S., e Mattsson, M. (2008). Systematic mapping studies in software engineering. In Proceedings of the 12th International Conference on Evaluation and Assessment in Software Engineering - EASE'08, pages 68-77, Swindon, UK. BCS Learning \& Development Ltd.

Reyes, J. A. (2015). The skinny on big data in education: learning analytics simplified. TechTrends, 59(2):75-80.

Rigo, S. J., Cambruzzi, W., Barbosa, J. L. V., e Cazella, S. C. (2014). Aplicações de mineração de dados educacionais e learning analytics com foco na evasão escolar: oportunidades e desafios. Revista Brasileira de Informática na Educação, 22(01):132146.

Rusu, C., Roncagliolo, S., Rusu, V., e Collazos, C. (2011). A methodology to establish usability heuristics. In The Fourth International Conference on Advances in ComputerHuman Interactions (ACHI 2011), pages 51-59, Guadalupe, França. ACM.

Tervakari, A. M., Silius, K., Koro, J., Paukkeri, J., e Pirttilä, O. (2014). Usefulness of information visualizations based on educational data. In 2014 IEEE Global Engineering Education Conference (EDUCON), pages 142-151, Istambul, Turquia. IEEE.

Vieira, C., Parsons, P., e Byrd, V. (2018). Visual learning analytics of educational data: a systematic literature review and research agenda. Computers and Education, 122:119135. 